Commonest cause of death was cardiac related events (24\%), followed by sepsis (23\%) or liver related complications (14\%). Disease duration prior to transplantation, initial presentation with autonomic rather than peripheral neuropathy, TTR mutation, and modified body mass index (mBMI) of $<600$, indicating poor nutritional status, were identified as significant factors influencing survival after LT $(\mathrm{p}<0.01)$.

Conclusion Liver transplantation is rational and effective treatment for FAP with excellent long-term outcomes and 10-year survival $>70 \%$. Type of mutation, nutritional status, disease duration and degree of autonomic involvement are significant prognostic factors.

\section{P73 PRELIMINARY RESULTS OF THE STUDY OF ACUTE LIVER TRANSPLANT (SALT): NSAID EXPOSURE AND RISK OF ACUTE LIVER FAILURE LEADING TO TRANSPLANTATION IN 7 EUROPEAN COUNTRIES}

doi:10.1136/gutjnl-2011-300857a.73

${ }^{1} \mathrm{D}$ Thorburn, ${ }^{2} \mathrm{E}$ Gulmez, ${ }^{2} \mathrm{~S}$ Lignot, ${ }^{3} \mathrm{D}$ Larrey, ${ }^{4} \mathrm{C}$ de Vries, ${ }^{5} \mathrm{~S}$ Perez-Gutthann, ${ }^{6} \mathrm{~J} \mathrm{~L} \mathrm{Montastruc,}{ }^{7} \mathrm{M}$ Sturkenboom, ${ }^{8} \mathrm{~J}$ Benichou, ${ }^{9} \mathrm{G}$ Velo, ${ }^{10} \mathrm{~A}$ Caputi, ${ }^{2} \mathrm{~F}$ Salvo, ${ }^{2} \mathrm{~F}$ Hamoud, ${ }^{2} \mathrm{~S}$ Micon, ${ }^{2} \mathrm{R}$ Lassalle, ${ }^{2} \mathrm{~J}$ Jove, ${ }^{3} \mathrm{G} \mathrm{P}$ Pageaux, ${ }^{11} \mathrm{Y}$ Horsmans, ${ }^{12} \mathrm{~J}$ Bernuau, ${ }^{13} \mathrm{~F}$ Bissoli, ${ }^{7} \mathrm{~B}$ Stricker, ${ }^{14} \mathrm{~A}$ Gatta, ${ }^{15} \mathrm{E}$ Monteiro, ${ }^{16} \mathrm{I}$ Vafiadis, ${ }^{17} \mathrm{~A}$ McCormick, ${ }^{7} \mathrm{H}$ Metselaar, ${ }^{7} \mathrm{E}$ Sen, ${ }^{4} \mathrm{~A}$ Nightingale, ${ }^{2} \mathrm{P}$ Blin, ${ }^{2} \mathrm{~N}$ Moore. ${ }^{1}$ Royal Free NHS Trust, Liver Unit; ${ }^{2}$ University Bordeaux Segalen, Bordeaux; ${ }^{3} \mathrm{CHU}$ St Eloi Hospital, Montpellier; ${ }^{4}$ Bath University, Bath; ${ }^{5}$ RTI Health Solutions, Barcelona; ${ }^{6} \mathrm{CHU}$ de Toulouse, Toulouse; ${ }^{7}$ Erasmus University MC, Rotterdam; ${ }^{8} \mathrm{CHU}$ de Rouen, Rouen; ${ }^{9}$ University of Verona, Verona; ${ }^{10}$ Policlinico Universitario, Messina; ${ }^{11}$ Louvain Catholic University, Louvain; ${ }^{12}$ Beaujon Hospital, Clichy; ${ }^{13}$ Clinica San Gaudenzio, Novara; ${ }^{14}$ Padua University Hospital, Padua; ${ }^{15}$ Santa Maria Hospital, Lisbon; ${ }^{16}$ Athens University School of Medicine, Laiko General Hospital, Athens; ${ }^{17}$ St. Vincent's University Hospital, Dublin

Introduction The risk of acute liver failure (ALF) related to NSAIDs is still discussed and the European Medicines Agency requested a study investigating this. University Bordeaux Segalen conducted the study independently.

Aim To estimate the incidence rates of ALF leading to registration for liver transplantation (LT) in patients exposed to NSAIDs.

Method Multinational, multicentre, case-population study performed in France, Greece, Ireland, Italy, the Netherlands, Portugal, and the UK retrospectively evaluating a 3-year period (2005-2007) in adults. Data of ALF cases were sought through liver transplant registries and hospital records. Demographic and clinical data were collected for all ALF cases and drug use information was collected for the exposure window of 30 days prior to index date (ID, initial symptoms of liver disease). For ALF cases exposed to NSAIDs (ATC code M01A), rate per million treatment-years (tt-yrs) was calculated using sales data from IMS. Poisson CIs (95\% CI) were estimated.

Results In the seven participating countries, 62 LT centres were identified and contacted, five were excluded (four paediatric, one oncology), and 50 actively contributed data before database lock. Among the 8824 patients identified from LT lists for the period 2005-2007, 500 were cases of ALF: 197 with identified clinical cause, 21 with incomplete or unavailable medical files, and 241 drugexposed without identified clinical cause. Among the latter, 34 were exposed to at least one NSAID, 123 exposed to other drugs, and 84 were acute drug intoxications. Mean age of NSAID-exposed ALF cases was 43.8 years, 24 were female. Event rates per million treatment-years were 4.4 (95\% CI 3.0 to 6.1) for all NSAIDs pooled, 5.6 (2.4 to 11.1) for nimesulide (8 cases), 5.8 (2.8 to 10.6) for ibuprofen (10 cases), 4.5 (1.5 to 10.4) for diclofenac (5 cases), and 4.7 (1.0 to 13.6) for ketoprofen ( 3 cases). 71 of the 157 non-intoxication cases had been exposed to paracetamol ( 9.8 per million treatment-years, $95 \%$ CI 7.7 to 12.4 ), and 83 of the 84 intoxications.

Conclusion In seven countries over 3 years only 34 NSAID-exposed ALF cases leading to registration for LT were identified with no differences in incidence rates per million tt-yrs among the most used NSAIDs. Non-overdose paracetamol-associated liver failure was twice more common.

\section{P74 HEPATOCYTE TRANSPLANTATION IN RATS WITH ACUTE LIVER FAILURE USING CELLS LABELLED WITH A CLINICAL GRADE MRI CONTRAST AGENT}

doi:10.1136/gutjnl-2011-300857a.74

${ }^{1} \mathrm{~J}$ Puppi, ${ }^{1} \mathrm{R}$ D Hughes, ${ }^{2} \mathrm{M}$ Modo, ${ }^{1} \mathrm{R}$ R Mitry, ${ }^{1} \mathrm{~A}$ Dhawan. ${ }^{1}$ Institute of Liver Studies, King's College London School of Medicine at King's College Hospital; ${ }^{2}$ Institute of Psychiatry, King's College London

Introduction Hepatocyte transplantation is being evaluated as an alternative to orthotopic liver transplant. However, the fate of hepatocytes after transplantation is not well defined.

Aim The aims of the study were to: (1) investigate the possibility of labelling hepatocytes in vitro using superparamagnetic iron oxide nanoparticles (SPIOs), (2) determine the effects of labelling on cell viability and function, and (3) perform in vivo experiments on tracking labelled cells by MRI.

Method Human and rat hepatocytes were labelled in culture for $16 \mathrm{~h}$ with clinical SPIOs $(12.5 \mu \mathrm{g} \mathrm{Fe} / \mathrm{ml})$ and protamine sulphate $(3 \mu \mathrm{g} / \mathrm{ml})$ as a transfection agent. Cellular iron uptake was determined using Prussian blue staining, and quantified by a ferrozine-based assay. Cell viability and function were assessed using LDH leakage, mitochondrial dehydrogenase activity, $\left[{ }^{14} \mathrm{C}\right]$-leucine incorporation, albumin and urea assays. Effects of labelled cells on T2-weighted images were assessed in vitro using a 7-T MR scanner. Intrasplenic transplantation of $2 \times 10^{7}$ male rat hepatocytes labelled with SPIOs $(n=4)$ or non-labelled $(n=4)$ was performed in female recipients $28-30 \mathrm{~h}$ after acute liver failure induction by intraperitoneal injection of $\mathrm{D}$-galactosamine. Hepatocytes were also marked with the fluorescent dye CM-DiI. A control group $(n=4)$ received medium injection only. T2*weighted gradientecho images at 7-T MRI were acquired at day 7 post-acute liver failure induction. Transplanted cells were detected in the liver by PCR for the Y-chromosome (Sry-2 gene) and histological analysis.

Results Mean intracellular iron concentrations were $11.4 \pm \mathrm{SE} 1.1 \mathrm{pg} /$ cell in human and $8.6 \pm 0.3 \mathrm{pg} / \mathrm{cell}$ in rat hepatocytes. Cell viability and metabolic function were not significantly affected at these SPIO concentrations. In vitro MRI of SPIO-labelled cells (2000 cells/ $\mu \mathrm{l})$ induced a $50 \%$ change in T2 relaxivity compared to non-labelled cells. SPIOs were detected in rat liver as a decrease in the MRI signal intensity 6 days after transplantation in the three survivors. On histology most of the SPIO particles were located in Kupffer cells, indicating the loss of iron oxide particles from hepatocytes. In keeping with this, labelled cells could not be detected in the liver by the fluorescent dye or by PCR for Sry-2 gene.

Conclusion Optimum conditions to label human and rat hepatocytes with SPIOs were determined, which did not affect cell viability or metabolic function, and were sufficient for in vitro MRI detection. However, the clearance of hepatocytes after transplantation limits the value of MRI for assessing long-term hepatocyte engraftment.

\section{P75 IMPAIRED CARDIORESPIRATORY RESERVE IN PRIMARY BILIARY CIRRHOSIS PATIENTS UNDERGOING LIVER TRANSPLANT ASSESSMENT}

doi:10.1136/gutjnl-2011-300857a.75

${ }^{1} \mathrm{~J}$ Prentis, ${ }^{2} \mathrm{D}$ E Jones, ${ }^{2} \mathrm{M}$ I Trenell, ${ }^{1} \mathrm{C}$ P Snowden. ${ }^{1}$ Freeman Hospital; ${ }^{2}$ Newcastle University

Introduction It has been previously shown that $\mathrm{PBC}$ patients have bioenergetic abnormality in both peripheral and cardiac muscle. In 
particular they exhibit significantly lower cardiac muscle phosphocreatine-to-ATP ratio (measure of cardiac bioenergetic integrity) compared with control subjects. In other disease settings, such as cardiac failure, changes of this type have been associated with impaired cardiac function and increased risk of cardiac death.

Aim The objective of the present study was to examine whether these changes are reflected in systemic measurements of cardiopulmonary reserve determined by a non-invasive cardiopulmonary exercise test (CPET).

Method Consecutive PBC patients being assessed for liver transplantation underwent CPET. The test was conducted in a consistent environment and reviewed by a trained physician to determine objective measures of cardiorespiratory reserve. A control group of consecutive patients with primary sclerosing cholangitis (PSC), also being assessed for liver transplantation was also tested. We compared the results of CPET of all patients with a diagnosis of PBC with those with PSC. Patient demographics and MELD scores at assessment were also collected. A non-paired t test was used to determine group differences.

Results In total, 38 patients had a diagnosis of either PBC or PSC. Three patients (2 PBC and 1 PSC) did not exercise sufficiently to gather meaningful results and were excluded from the analysis. The PSC patients assessed for transplantation had significantly worse liver disease as assessed by the MELD score. However, all measures of cardiorespiratory reserve derived from CPX testing were significantly lower in the PBC group. There was no statistical difference between the two groups with respect to age, thus excluding age as the underlying factor in decreasing their fitness.

Conclusion In this cohort, patients with $\mathrm{PBC}$, despite having lower MELD scores and equivalent age at transplantation assessment, had significantly impaired cardiorespiratory reserve, when compared to patients with PSC. The results add to the evidence that there is a specific PBC-related bioenergetic effect due to the immunology of $\mathrm{PBC}$ that is absent in PSC. This finding could have significant relevance on both future studies and treatment regimes to improve cardiovascular fitness.

\section{Abstract P75 Table 1}

\begin{tabular}{llll}
\hline & PBC & PSC & p Value \\
\hline Number & 24 & 11 & \\
Age yrs mean (SD) & $56.1(8.9)$ & $56.1(12.9)$ & 0.999 \\
MELD mean (SD) & $13.0(6.60)$ & $18.8(4.15)$ & 0.004 \\
AT mean (SD) & $10.7(2.8)$ & $13.3(3.0)$ & 0.017 \\
Peak V0 ${ }_{2}$ mean (SD) & $13.6(3.4)$ & $17.9(4.7)$ & 0.004 \\
OEUS/kg mean (SD) & $18.7(4.5)$ & $22.8(4.5)$ & 0.018 \\
V02/HR mean (SD) & $7.6(2.7)$ & $9.7(1.5)$ & 0.022 \\
\hline
\end{tabular}

P76 META-ANALYSIS OF PUBLISHED EVIDENCE SUPPORTS USE OF KING'S COLLEGE CRITERIA OVER MODEL FOR END STAGE LIVER DISEASE IN OUTCOME PREDICTION IN ACUTE LIVER FAILURE

doi:10.1136/gutjnl-2011-300857a.76

${ }^{1}{ }^{2} \mathrm{M}$ J W McPhail, ${ }^{2} \mathrm{~N}$ Senvar, ${ }^{3} \mathrm{~J}$ A Wendon, ${ }^{3} \mathrm{~W}$ Bernal. ${ }^{1}$ Institute of Liver Studies, King's College Hospital, Denmark Hill, London, UK; ${ }^{2}$ Liver \& Anti-viral Centre, St Mary's Hospital, South Wharf Street, Imperial College London, UK; ${ }^{3}$ Institute of Liver Studies, King's College Hospital, Denmark Hill, London, UK

Introduction Outcome prediction is a cornerstone of the management of Acute Liver Failure (ALF) where Emergency Liver Transplantation (ELT) is indicated for predicted death. The King's College Criteria (KCC) for paracetamol overdose (POD) and non-POD ALF are the benchmark prognostic scores but recent reports have suggested the Modified End Stage Liver Disease (MELD) score could replace KCC.
Aim To meta-analyse and compare diagnostic performance for outcome prediction of KCC and MELD in ALF.

Method A systematic database search was performed and retrieved articles graded according to a pre-agreed proforma of methodological quality. Collated data were meta-analysed for summary sensitivity, specificity, Diagnostic OR, DOR meta-regression and ROC curve analysis. Pre-specified subgroup analysis was performed on the basis of methodological quality, the severity of hepatic encephalopathy (HE) of reported patients, and exclusion of those who underwent ELT.

Results 32 studies published between 1992 and 2009 with data on 3008 patients (2464 from KCC and 544 MELD) were available for production of $2 \times 2$ tables. Taking data where transplanted patients were excluded summary sensitivity for KCC was 63 (95\% CI 60 to 66) \%, specificity 91 (90 to 93) \% and DOR 18 (8.9 to 37). Summary sensitivity for MELD was $82(95 \%$ CI 77 to 86$) \%$, specificity 65 (59to71) \% and DOR 11 (5.3 to 23; RDOR 0.75 (0.18 to 3.13))). Despite different MELD cut-offs between studies no statistical evidence of threshold was found and the AUROC for MELD was 0.83 (SE 0.05) and 0.88 (0.03) for KCC. A lack of patient level data prevented statistical comparison between these areas. The DOR for KCC in POD ALF was 27 (9-83) and 13 (5-31) in non-POD ALF. Heterogeneity (using the $\mathrm{I}^{2}$ statistic) in the DOR for MELD was $49 \%$ and $80 \%$ for KCC although this was not dependent on aetiology. The lower sensitivity for KCC could be overcome by dynamic application of the criteria.

Conclusion MELD is not superior to KCC on the basis of quantitative analysis of published evidence. While both scores may give complementary sensitivity and specificity (particularly in cases where bilirubin may have more prognostic value) the DOR and AUROC superiority of KCC suggest they should remain the preferred method of outcome prediction and listing decision for ELT in ALF, particularly in cases of POD-ALF.

\section{P77 A PHARMACIST DELIVERED STRATIFIED CONVERSION PROTOCOL FROM HEPATITIS B IMIMUNOGLOBULIN (HBIG) TO TENOFOVIR OR ENTECAVIR IS EFFICACIOUS, SAFE AND COST-EFFECTIVE FOR PREVENTION OF RECURRENCE OF HEPATITIS B VIRUS (HBV) IN LIVER TRANSPLANT (LT) RECIPIENTS}

doi:10.1136/gutjnl-2011-300857a.77

${ }^{1} \mathrm{~S}$ Knighton, ${ }^{2} \mathrm{~K}$ Agarwal, ${ }^{2} \mathrm{M}$ Heneghan, ${ }^{2} \mathrm{~J}$ O'Grady, ${ }^{2} \mathrm{M}$ Bruce, ${ }^{2} \mathrm{C}$ Carey, ${ }^{2} \mathrm{~N}$ Heaton, ${ }^{2}$ A Suddle. ${ }^{1}$ Pharmacy Department and Institute of Liver Studies and Transplantation, King's College Hospital NHS Foundation Trust, London, UK; ${ }^{2}$ Institute of Liver Studies and Transplantation, King's College London School of Medicine at King's College Hospital NHS Foundation Trust, London, UK

Introduction The use of HBIG based prophylactic regimensto prevent recurrence of HBV in patients who have undergone LT is highly efficacious and well documented. However the long-term administration of HBIG can be time consuming, costly and inconvenient for the patient. With the advent of more potent oral anti$\mathrm{HBV}$ agents the optimal long-term prophylactic strategy remains debatable.

Aim A prospective single centre experience of switching from an intra-muscular (IM) HBIG based regimen to monotherapy tenofovir (TDF) or entecavir (ETV) to prevent HBV recurrence post LT.

Method Patients receiving HBIG based prophylactic regimens were referred to a Pharmacist led clinic. Those with no serological evidence of HBV recurrence were considered for switch to monotherapy TDF or ETV. Decisions were based on clinical assessment and renal function, following an agreed stratified protocol. Data reported is an interim analysis 6 months post-switch. All results are presented as median. 